\title{
Editorial
}

\section{The health of nurses: Political rhetoric or a valid concern?}

\author{
Rebecca Jester \\ Professor of Nursing, Institute of Health, University of Wolverhampton, West Midlands, UK
}

In recent years there has been an increasing focus on health care professionals being healthy and in England this is affirmed in The NHS Five Year Forward View which asks all employees to "stay healthy, and serve as health ambassadors" (NHS England, 2014, p.11). Specifically, nurses appear to have been singled out regarding their own personal health and their duty to act as healthy role models. The International Council of Nurses (ICN) has called for nurses to make "a personal commitment to eat healthily, exercise appropriately, drink sensibly and avoid the use of tobacco" (ICN, 2010, p. 37). The NMC in the UK advises nurses to recognise that their behaviour at all times can affect and influence others (NMC, 2015). In England there is increasing concern regarding obesity levels amongst nurses and other healthcare staff, with a cross-sectional survey by Kyle et al. (2017) reporting obesity prevalence was high across all occupational groups including: among nurses $(25.1 \%)$; other healthcare professionals (14.4\%); non-health-related occupations (23.5\%); and unregistered care workers who had the highest prevalence of obesity $(31.9 \%)$.

The policy discourse to date seems to exclusively focus on physical health, to the detriment of discussing health in a more holistic context including mental and psychological health of nurses and other healthcare staff. There also appears to be criticism toward nurses who are not manifestly healthy.

The aim of this editorial is to discuss if there is sufficient evidence to suggest that nurses should be healthy role models, if nurses' health impacts on patients' health behaviours and what can, and should individual nurses and employers do to promote the health of nurses. A recent Delphi study in England (Kelly et al., 2017) explored the views of stakeholders (nurses, student nurses, service users, nurse educators and policy makers) regarding the expectation that nurses should be role models for healthy behaviours and reported that opposing the view that nurses should epitomise and demonstrate healthy behaviours to support patients and be credible in advice, stakeholders agreed it was more important to be seen as "human" and understand the challenges of health behaviour change.

Email address: rfjester@hotmail.co.uk (R. Jester)
Successful health promotion advice which results in sustained changes in health behaviours is a complex process and dependent on an individual's readiness to change, not simply the imitation of an outwardly healthy-looking nurse! That said the service users in this study raised the issue of credibility of nurses giving advice about health when they themselves looked unhealthy (obese, smell of smoking etc).

So, let us consider why is the prevalence of obesity in nurses higher than in non-health care professionals and indeed other health care professionals (Kyle et al., 2017). There is an increasing realisation that the workplace and working conditions of many nurses is inherently unhealthy, with irregular hours, lack of regular meal and rest breaks, lack of access to healthy food options and increasing stress as nurses try to provide high quality care whilst facing inadequate staffing levels and skill mix. Public Health England estimates the cost to the NHS of staff absence due to poor health at $£ 2.4 \mathrm{bn}$ a year, in response to this a CQUIN on NHS health and wellbeing became operational in April 2016 (NHS, 2016), which includes introduction of health and wellbeing initiatives into the workplace, healthy food for staff, visitors and patients and increasing uptake of flu vaccinations by frontline staff. However, I would suggest this CQUIN is insufficient to support nurses' health. If nurses are unable to take regular breaks, and or leave the ward/department to access the new healthy food options or health and wellbeing initiatives it is not going to make a difference.

In my role as Adjunct Professor at the University of Southern Denmark I make several visits a year to spend time with colleagues in the orthopaedic department at Zealand Universitetshospital, where every departmental manager is allocated a budget to spend on staff well-being, some managers use this to provide a large box of fresh fruit for staff every week. Also nursing staff have access to a comfortable staff room situated in the ward/department, with a small kitchen and dining table and sit and eat their meals together (most of the time). I have noticed this gives them opportunity to also talk about their concerns with each other and to provide collegial support. I always come away from Denmark thinking the Danish get a lot of things right, they do pay high rates of tax, but it seems to be very well spent! 
I hope this editorial will stimulate some debate and we would really like to hear about any workplace initiatives or ideas you have about promoting the health of nurses and other health care workers.

\section{References}

International Council of Nurses, 2010. Delivering Quality, Serving Communities: Nurses Leading Chronic Care. Geneva, Switzerland. Available at: http://www.icn. ch/indkit.htm.

Kelly, M., Wills, J., Jester, R., Spellar, V., 2017. Should nurses be role models for healthy lifestyles? results from a modified Delphi study. J. Adv. Nurs. 73 (3), $62-77$
Kyle, R., Wills, J., Mahoney, C., Hoyle, L., Kelly, M., Atherton, 2017. Obesity prevalence among healthcare professionals in England: a cross-sectional study using the Health Survey for England. BMJ Open https://doi.org/10.1136/ bmjopen-2017-018498.

NHS England, 2014. Care Quality Commission, Health Education Engalnd, Monitor, Public Health England and Trust Development Authority. NHS England, London, NHS five year forward view.

NHS England, 2016. NHS Staff Health \& Wellbeing: CQUIN guidance. NHS England, London, UK.

Nursing and Midwifery Council, 2015. Character and Health-decision Making Guidance, available at:http://www.nmc.org.uk/globalassets/sitedocuments/registration/ character-and-health-decision-making-guidance.pdf. 